\title{
Shear-driven density segregation: an experimental study
}

\author{
Monica Tirapelle ${ }^{1}$, Andrea C. Santomaso ${ }^{1 *}$, Patrick Richard ${ }^{2}$, and Riccardo Artoni ${ }^{2}$ \\ ${ }^{1}$ APTLab - Advanced Particle Technology Laboratory, Department of Industrial Engineering, University of Padova, Italy \\ ${ }^{2}$ MAST-GPEM, University Gustave Eiffel, IFSTTAR, F-44344 Bouguenais, France
}

\begin{abstract}
Granular materials can segregate spontaneously due to differences in particle properties when subjected to vibrations, shear strain or because of the equipment geometries. Although the difference in particle size is the most critical factor that drives segregation, the effects of large density difference may also be detrimental for a lot of industries. In this work, we experimentally investigate density-driven segregation in bi-disperse mixtures of particles having the same size but different density when subjected to non-uniform shear rates. We found that the features of the segregation process are related to the density ratio as well as to the dimensionless loaded mass. The experimental outcomes are then compared with the solution of a simple density-driven segregation model. The model can successfully capture the main features of segregation driven by density for a range of density ratios.
\end{abstract}

\section{Introduction}

Granular materials composed of particles with different sizes, densities, shapes, or surface properties may experience unexpected segregation when subjected to process vibration or shear [1]. Even though the particle size is known to be the most relevant factor determining segregation, the effect of large density differences may also be of importance for some industries. Indeed, because of density driven segregation, lighter particles are likely to rise whereas heavier particles sink to the bottom, and the mixture un-mixes [2].

In this work we focus on segregation driven by density in binary mixtures of equal sized particles under nonuniform shear rates. The experiments are conducted in an annular shear cell with a rotating bottom and an overloaded top wall similar to that used in previous published works [3-6]. Herein, the local horizontal velocity profile decays exponentially, leading in turn to an exponential shear profile. At the beginning, the lower half of the cell is filled with the lighter component, whereas the heavy one is on top. Under shear, the system progresses through the reverse segregated configuration. The system is filmed through the transparent sidewall, and the concentration profiles are evaluated by means of postprocessing image analysis. We found that segregation driven by density is strictly related to the shear localization features.

To model segregation by density, we implemented a segregation velocity based on a modified version of the Stokes' law within a 1-dimensional segregation-diffusion transport equation that tracks the evolution of the specie volume fraction. To close the model, constitutive relations for granular viscosity and friction coefficient were then introduced. The solutions of the model are comparable with the experimental outcomes. The model can reasonably predict density-driven segregation for a range of density ratios.

\section{Materials and method}

\subsection{Experimental setup and conditions}

Experiments were performed using binary mixtures of equal sized spherical particles having different intrinsic density. The mixtures composition was $50 / 50$ by volume. The particles had mean diameter, $d_{p}$, equal to $\approx 6 \mathrm{~mm}$ and were made of polypropylene $\left(\rho=0.95 \mathrm{~g} / \mathrm{cm}^{3}\right)$, filled polyoxymethylene $\left(\rho=1.75 \mathrm{~g} / \mathrm{cm}^{3}\right)$, glass $(\rho=$ $\left.2.45 \mathrm{~g} / \mathrm{cm}^{3}\right)$ and ceramic $\left(\rho=3.50 \mathrm{~g} / \mathrm{cm}^{3}\right)$. Owing to the differences in colour and/or brightness of each component, we could quantify the local amount of each specie by image analysis. Table 1 lists the mixtures we employed, their corresponding density ratios $\left(\delta_{R}=\right.$ $\left.\rho_{H} / \rho_{L}\right)$ and the dimensionless loading mass $\widetilde{M}$ defined as the loading mass divided by the mass of grains in the system $\left(\widetilde{M}=M_{w} / M_{g}\right)$. The latter is important because it influence the shear thickness.

Our experimental apparatus was an annular shear cell with a rotating bottom wall. The vertical walls were two transparent coaxial cylinders made of polymethylmethacrylate (PMMA). The smallest cylinder had outer diameter equal to $90 \mathrm{~mm}$, whereas the largest cylinder had inner diameter of $190 \mathrm{~mm}$. Thus, the thickness of the annular region was $50 \mathrm{~mm}$ wide. For the no-slip condition to hold, the bottom wall was 3D printed in PLA with bumpiness. The bumpiness is characterized by hemispheres having diameter equal to $d_{p}$ and arranged in a triangular mesh with a mean distance of $2 d_{p}$ between their edges. The bottom wall was then screwed to a rotating plate, driven by a stepper motor. The top wall, again printed in PLA with bumpiness, was free to move vertically but it could not rotate. On it, different loading forces could be applied. All the experimental results reported in this paper have been obtained with the bottom

* Corresponding author: andrea.santomaso@unipd.it

A video is available at https://doi.org/10.48448/aae5-9m67 


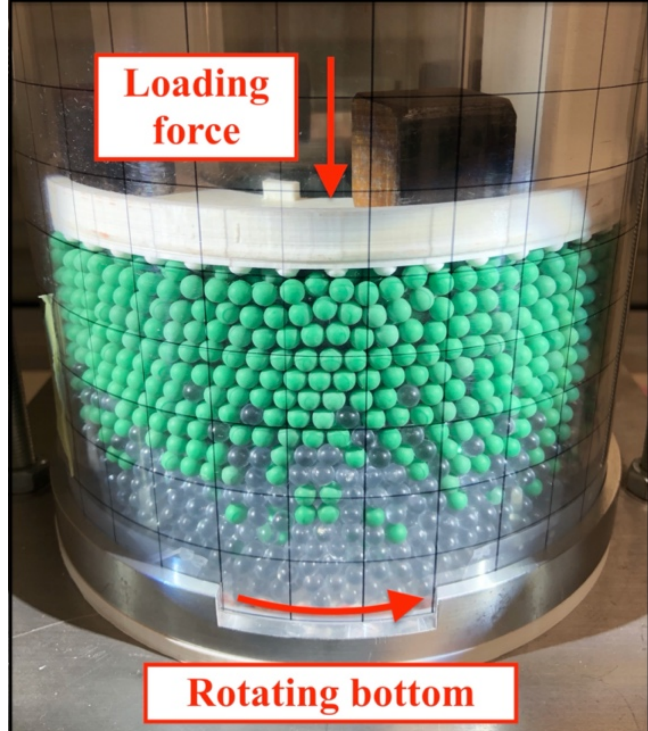

Fig. 1. Photograph of the experimental setup. The annular shear cell is characterized by a rotating bumpy bottom and a loaded top wall that provides confinement. The system is filled with a 50/50 mix of grains by volume.

plate rotating at a constant angular velocity of $\Omega=$ $23.44 \mathrm{rpm}$ and with $1.093 \mathrm{~kg}$ of load applied on the top wall (i.e. weight of the top wall and additional steel weights). The experimental set-up is shown in Fig. 1. This type of geometry has numerous advantages: the generated granular flow is continuous, there is no need to feed the system while it is running, the initial and final states of the experiments can be well defined, and the shear rate is not uniform [5]. To characterize the shear rate, we were primarily interested in the horizontal velocity profile along the direction of gravity, $u(z)$. According to May et al. [5] and Artoni et al. [6], the horizontal velocity profile developed within an annular shear cell with rotating bottom can well described by an exponential function of the form:

$$
u(z)=u_{1}+\left(u_{0}-u_{1}\right) e^{-\frac{z}{\delta}},
$$

where $u_{0}$ is the velocity of the bottom wall at $z=0, u_{1}$ is the slip velocity at the top boundary, and $\delta$ is the coefficient of exponential decay. Owing to the exponential decay of $u(z)$, the shear rate, $\dot{\gamma}$, is obviously not uniform throughout the domain. Indeed $\dot{\gamma}$ is, by definition, the gradient of the velocity in the direction perpendicular to the flow $(\dot{\gamma} \equiv \partial u / \partial z)$. Furthermore, because of the sidewall friction and the applied load, the shear is localized at the bottom [6].

Initially, the system is stratified: the lower half of the cell is filled with the lighter material, whereas the heavier

Table 1. Bi-dispersed mixtures employed.

\begin{tabular}{|c|c|c|}
\hline Combination & $\boldsymbol{\delta}_{\boldsymbol{R}}$ & $\widetilde{\boldsymbol{M}}$ \\
\hline Glass-Filled POM & 1.40 & 0.44 \\
\hline Filled POM-Polypropyene & 1.85 & 0.68 \\
\hline Glass- Polypropyene & 2.59 & 0.54 \\
\hline Ceramic-Polypropyene & 3.70 & 0.41 \\
\hline
\end{tabular}

grains are located on an upper layer. The total amount of the grains filled in the system corresponds to a bed height of $90 \mathrm{~mm}$, namely 15 particles diameters. Under shear, the particles mix up and then re-segregate again until the stable configuration is reached.

The segregation process is recorded at the outer transparent sidewall with a GoPro Hero 4 black. Thus, information on component and position of each particle were obtained through post-processing image analysis. The images were first undistorted and cropped to obtain an investigation window $9 d_{p}$ wide and $15 d_{p}$ height. Then, the position of each single particle was detected thanks to the Hough Circles tool [7]. To discern the particle component, we performed threshold segmentation in the HSV color space since the HSV color space allows visual difference between components to be identified. With all this information, we could reconstruct the temporal evolution of the volume fraction of each specie at the sidewall and through the height of the cell.

\subsection{Theoretical model}

Since in our system the variables change predominantly along the direction of gravity, we developed a 1dimensional density driven-segregation model. In developing the model, we assumed for simplicity that the horizontal velocity profile is constant in time and independent of the local concentration.

To see how the volume fraction of the $i$-th component of the mixture, $v_{i}$, evolves in time and space, we employed the following diffusive-segregating transport equation:

$$
\frac{\partial v_{i}}{\partial t}=-\frac{\partial v_{i} w_{s, i}}{\partial z}+\frac{\partial}{\partial z}\left(\mathcal{D} \frac{\partial v_{i}}{\partial z}\right)
$$

where $\mathcal{D}$ is the diffusion coefficient and $w_{s, i}$ is the segregation velocity. The magnitude of the self-diffusion coefficient is proportional to the local shear rate and the square of the particle diameter [8]: $\mathcal{D} \propto \dot{\gamma}{d_{P}}^{2}$. Since our particles differ only by density, we assumed that their selfdiffusivity is the same. As segregation velocity, we used

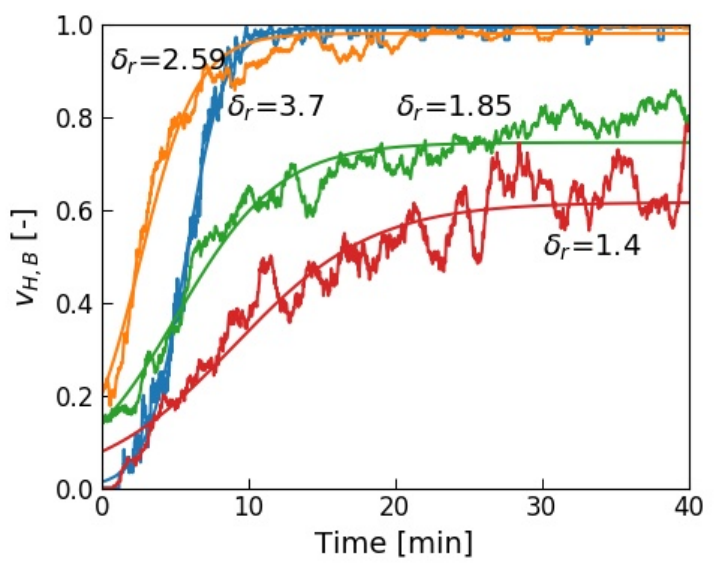

Fig. 2. Evolution of the volume fraction of the heavy component in the bottom layer of the cell for four different density ratios. Their fitting is done with a hyperbolic tangent function. 
a formulation analogue to the Stokes' law [9, 10]. Therefore, if a spherical particle of density $\rho_{i}$ and size $d_{p}$ fall in a bed of grains, its segregation velocity reads:

$$
w_{s, i}=k\left[\frac{g d_{p}^{2}}{18 \eta_{\operatorname{mix}}}\left(\rho_{i}-\rho_{\text {mix }}\right)\right]
$$

Here $k$ is a corrective factor; whereas $\rho_{\text {mix }}$ and $\eta_{\text {mix }}$ are the effective density and the effective viscosity of the solid mixture respectively:

$$
\begin{gathered}
\rho_{\text {mix }}=\sum_{i=1}^{n} \rho_{i} v_{i}, \\
\eta_{\text {mix }}=\frac{\mu(I) P}{|\dot{\gamma}|} .
\end{gathered}
$$

The latter is related to the overburden $P$ and the friction coefficient $\mu(I)$, which in turn is a function of the inertial number:

$$
I=d_{p}|\dot{\gamma}| \sqrt{\frac{\rho_{m i x}}{P}}
$$

As friction law, we used the one proposed by Jop et al. [11]:

$$
\mu(I)=\mu_{\min }+\frac{\mu_{\min }-\mu_{2}}{I_{0} / I+1} .
$$

This model relies on one free parameter: the corrective factor $k$. The parameters $\delta, u_{0}$ and $u_{1}$ appearing in the velocity profiles can instead be measured experimentally. All the results reported in this paper were obtained with $k=1.43$ and $\delta=0.18$.

\section{Results and discussion}

We evaluated density-driven segregation in binary mixtures of $50 \%$ initial volume fraction of each component and for the following density ratios: 1.40 , $1.85,2.59$ and 3.70. Fig. 2 shows the evolution of the volume fraction of the heavy grains at the bottom layer of the cell, $v_{H, B}$. In the case of higher density ratios, segregation dominates over diffusion. The bottom layer of the cell, which at the beginning is full of light particles, becomes at steady state almost all full of heavy grains. Furthermore, the final steady state is generally reached faster for greater $\delta_{R}$. With the decrease of $\delta_{R}$, the diffusional mixing competes always more against segregation. In the limiting case of $\delta_{R}$ approaching one (i.e. when the two components have the same density), the system evolves toward the perfectly mixed state and $v_{H, B}$ reaches the $50 \%$. Note that, some curves seem to start off with non-zero volume fraction: even if the initial condition was always the same, some heavy particles reached the bottom almost instantaneously. The experimental curves reported in Fig. 2 are fitted with a hyperbolic tangent function. This fitting allowed us to determine $v_{H, B}^{\infty}$ at steady state and the time required to

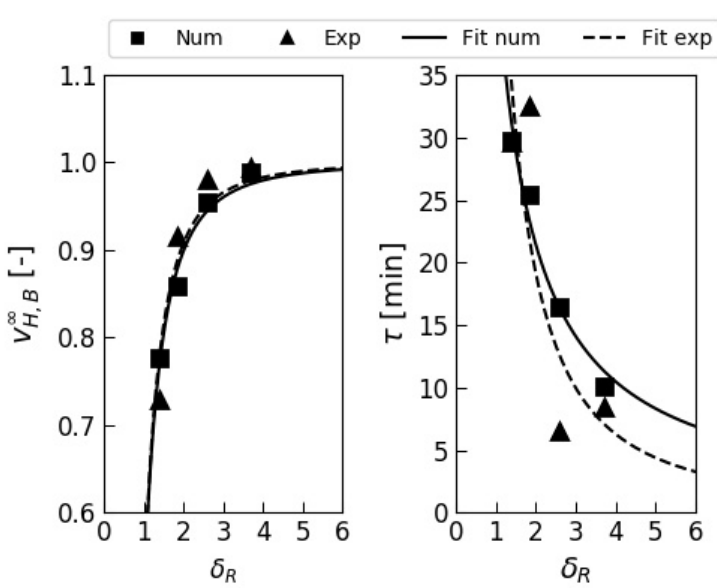

Fig. 3. On the left: the final volume fraction of the heavy component, $v_{H, B}^{\infty}$, evaluated at the bottom layer of the cell. On the right: the time required to reach the $88 \%$ of $v_{H, B}^{\infty}$. Square and triangular markers indicate numerical and experimental results, respectively. Furthermore, they are fitted with power functions and represented as solid lines and dashed lines respectively. In the case of $v_{H, B}^{\infty}$, numerical and experimental fittings coincide perfectly.

reach the $88 \%$ of $v_{H, B}^{\infty}$, namely $\tau$. The values of $v_{H, B}^{\infty}$ and $\tau$ are reported in Fig. 3 as a function of the density ratio. In the same figure we have also reported $v_{H, B}^{\infty}$ and $\tau$ obtained by solving the segregation model. If the prediction of $v_{H, B}^{\infty}$ is quite accurate, there are some discrepancies in the prediction of $\tau$. However, these discrepancies can be clearly explained. To solve the model, we assumed a constant velocity profile although this is not entirely true in reality. Furthermore, we implemented the same degree of exponential decay for each mixture even though $\delta$ is sensitive to $\widetilde{M}$. As shown
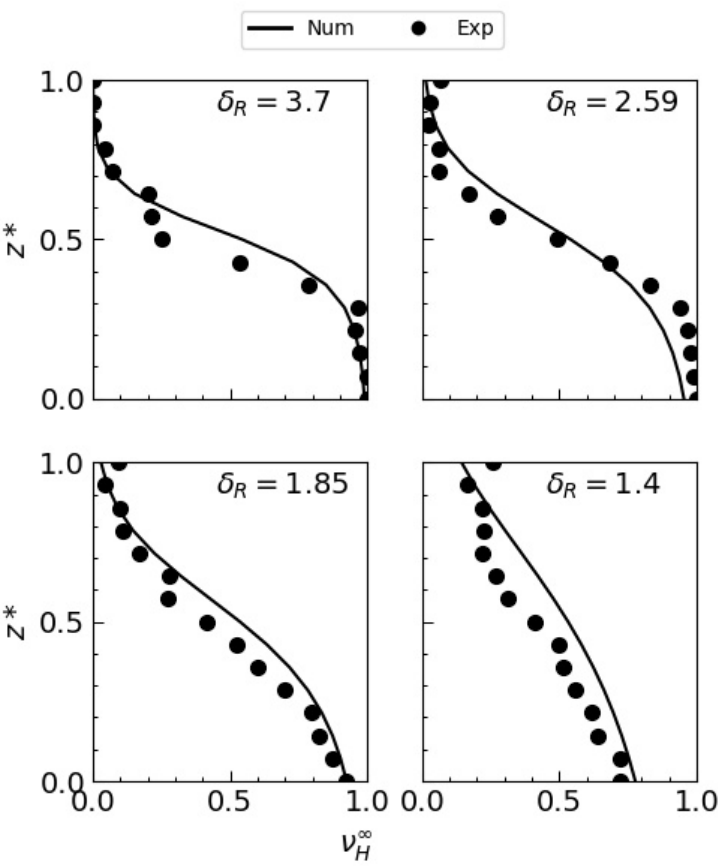

Fig. 4. The fully developed volume fraction profile for the four density ratios tested at steady state. The points have been obtained experimentally, whereas the solid lines represent the profile predicted by the model. 

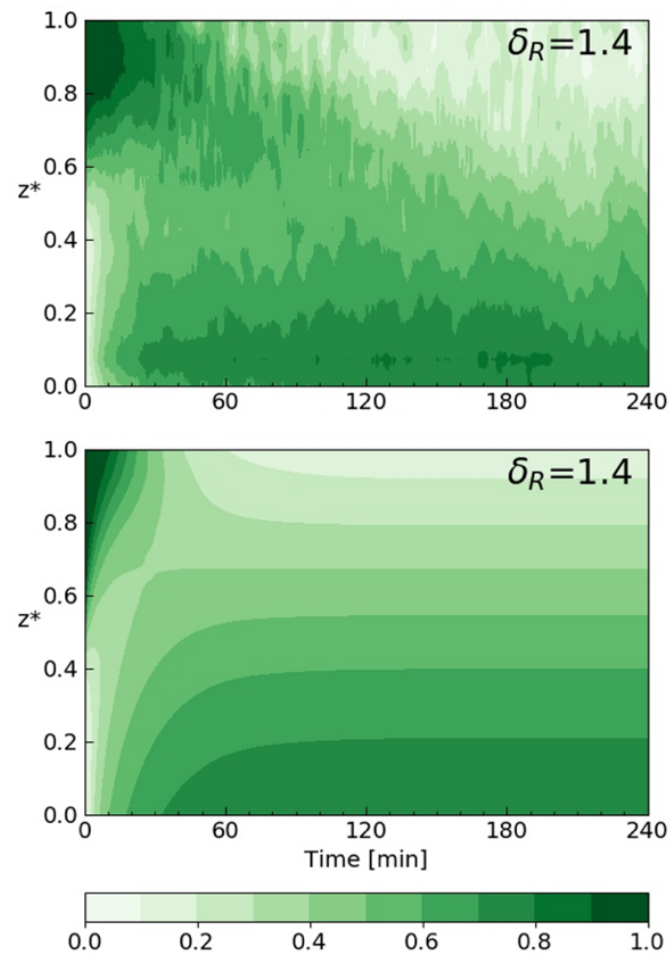

Fig. 5. Experimental measurement (on top) and a numerical simulation (on bottom) of the heavy particle concentration distribution for $\delta_{R}=1.40$.

in [6], the smaller the $\widetilde{M}$, the thinner has to be the shear band and vice versa.

In Fig. 4 we compare the fully developed profile of $v_{H}^{\infty}$ along $Z^{*}$ (i.e. the dimensionless cell height) obtained experimentally with the one predicted by the model. As it is possible to see, our density-driven segregation model can well capture the main features of segregation: the profile of $v_{H}^{\infty}$ is $\mathrm{S}$-shaped and smooth; moreover, smoothness is higher for smaller density ratios where diffusional mixing gains more importance over segregation. Fig. 5 and Fig. 6 display the evolution of the heavy grains volume fraction, $v_{H}$, in space and time for $\delta_{R}=1.4$ and $\delta_{R}=1.85$. In both cases, the experimental outcomes are compared with an equivalent numerical solution of the theory to prove the capabilities of the model. It is clear that, the model can reasonably predict density-driven segregation for a range of density ratios.

\section{Conclusion}

In this paper we have experimentally investigated density driven segregation under non uniform shear rates. The experimental outcomes have been compared with the analytical solutions of a density-driven segregation model. The results are consistent. The model has huge potential: it is simple, it requires low computational cost and it can well capture the main features of segregation for a range of density ratios.

Despite these advantages, the model is not without drawbacks. Firstly, it relies on a fitting parameter $k$ whose physical meaning is not clear. Nevertheless, we believe that $k$ is strictly related to the density ratio and the dimensionless loading mass. Furthermore, in order to
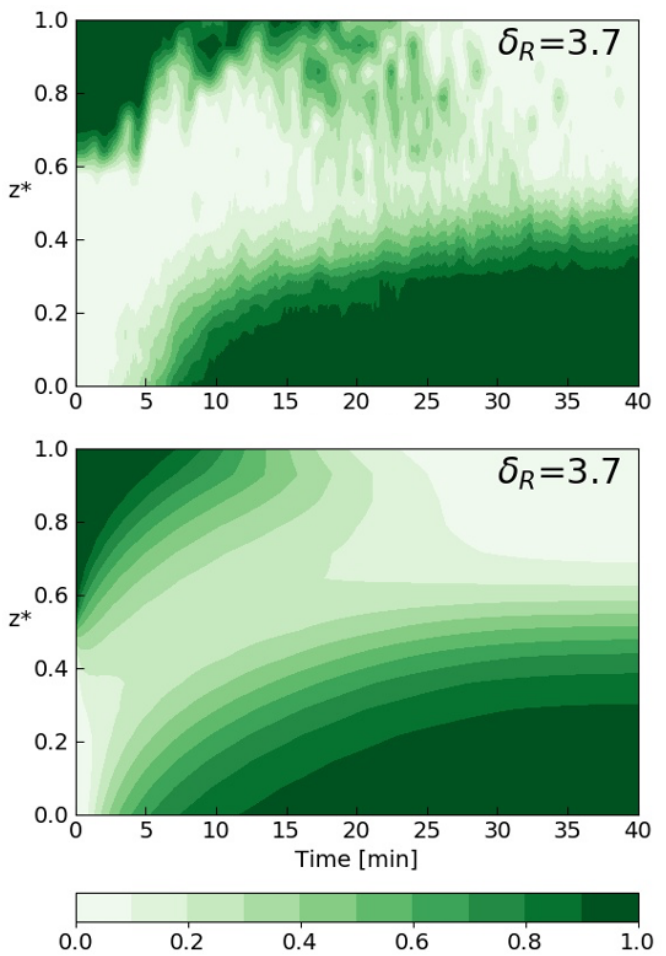

Fig. 6. Experimental measurement (on top) and a numerical simulation (on bottom) of the heavy particle concentration distribution for $\delta_{R}=3.70$.

implement the model, one should know the velocity profile, or the profile of the shear rate, within the system under investigation. The introduction of a non-local rheology instead of a prescribed flow field may help to overcome this limitation.

\section{References}

[1] J.M.N.T. Gray, Annu. Rev. Fluid Mech. 50, 407 (2018)

[2] D.V. Khakhar, J.J. McCarthy, J.M. Ottino, Phys. Fluids 9, 3600 (1997)

[3] S. Savage, M. Sayed, J. Fluid Mech. 142, 391 (1984)

[4] R. Wildman, T. Martin, J. Huntley, J. Jenkins, H. Viswanathan, X. Fen, D. Parker, J. Fluid Mech. 602, 63 (2008)

[5] L.B. May, L.A. Golick, K.C. Phillips, M. Shearer, K.E. Daniels, Phys. Rev. E 81, 051301 (2010)

[6] R. Artoni, A. Soligo, J.M. Paul, P. Richard, J. Fluid Mech. 849, 395 (2018)

[7] https://github.com/opencv/opencv

[8] B. Utter, R.P. Behringer, Phys. Rev. E 69, 031308 (2004)

[9] R.B. Bird, W.E. Stewart, E.N. Lightfoot, Transport Phenomena, revised 2 nd edition (2006)

[10]A. Tripathi, D.V. Khakhar, J. Fluid Mech. 717, 643 (2013)

[11]P. Jop, Y. Forterre, O. Pouliquen, Nature 441, 727 (2006) 